\title{
M-Polynomials and Degree-Based Topological Indices of the Molecule Copper(I) Oxide
}

\author{
Faryal Chaudhry, ${ }^{1}$ Iqra Shoukat, ${ }^{1}$ Deeba Afzal $\left(\mathbb{D},{ }^{1}\right.$ Choonkil Park $\left(\mathbb{D},{ }^{2}\right.$ Murat Cancan $\left(\mathbb{D},{ }^{3}\right.$ \\ and Mohammad Reza Farahani $\mathbb{i D}^{4}$ \\ ${ }^{1}$ Department of Mathematics and Statistics, University of Lahore, Lahore 54000, Pakistan \\ ${ }^{2}$ Research Institute for Natural Sciences, Hanyang University, Seoul 04763, Republic of Korea \\ ${ }^{3}$ Faculty of Education, Van Yüzüncü Yıl University, Van 65080, Turkey \\ ${ }^{4}$ Department of Applied Mathematics, Iran University of Science and Technology (IUST), Narmak, Tehran 16844, Iran
}

Correspondence should be addressed to Choonkil Park; baak@hanyang.ac.kr and Mohammad Reza Farahani; mrfarahani88@ gmail.com

Received 4 December 2020; Revised 20 December 2020; Accepted 28 January 2021; Published 24 February 2021

Academic Editor: Teodorico C. Ramalho

Copyright (C) 2021 Faryal Chaudhry et al. This is an open access article distributed under the Creative Commons Attribution License, which permits unrestricted use, distribution, and reproduction in any medium, provided the original work is properly cited.

\begin{abstract}
Topological indices are numerical parameters used to study the physical and chemical residences of compounds. Degree-based topological indices have been studied extensively and can be correlated with many properties of the understudy compounds. In the factors of degree-based topological indices, M-polynomial played an important role. In this paper, we derived closed formulas for some well-known degree-based topological indices like first and second Zagreb indices, the modified Zagreb index, the symmetric division index, the harmonic index, the Randić index and inverse Randić index, and the augmented Zagreb index using calculus.
\end{abstract}

\section{Introduction}

1.1. Application Background. A graph that represents the construction of a molecule and also their connectivity is known as a molecular graph, and such a representation is generally known as topological representations of molecule. Molecular graphs are normally characterized by means of exclusive topological basis for parallel of chemicals shape of a molecule with organic, chemical, or bodily homes. Study of graph has some programs of various topological indices in quantitative structure-activity relationship (QSAR) and quantitative structure-property relationship (QSPR), digital screenings, and computational drug designing citations as shown in $[1,2]$. Thus far, several exclusive topological indices have been established, and maximum of them are most effective graph descriptors in $[3,4]$; apart, some indices have proven their parallel with organic, chemical, or physical residences of secure molecules in [5-17].

In the field of mathematics, any graph has vertices and edges that are represented by the atoms and chemical bonds.
Graph that represents the construction of molecules and their connectivity is known as a molecular graph, and such representation is usually referred as topological representation of molecules. There are some significant topological indices like distance-based topological indices, degree-based topological indices, and primarily based topological indices. Among these works, distance primarily based topological indices unit works out a crucial task in a chemical graph started, specifically in chemistry $[18,19]$. Many fields have many features that can be solved with the help of graphs. In the physiochemical compounds or network systems, we have a tendency to abstractly outline exclusive ideas in modeling of mathematics. We have a tendency to refer to as the distinctive names, such as Randić index and national capital index.

A topological index is a numerical parameter of a graph and describes its topology. It describes the molecular shape numerically and is applied within the advancement of qualitative structure-activity relationships (QSARs). The following are the 3 types of topological indices: 
(1) Degree-based.

(2) Distance-based.

(3) Spectral-based.

Degree-based topological indices were studied extensively and may be correlated with many residences of the understudy molecular compounds. There is a strong relationship among distance-based and degree-based topological indices in [20]. Most commonly known invariants of such kinds are degree-based topological indices. These are actually the numerical values that correlate the structure with various physical properties, chemical reactivities, and biological activities. Topological indices are sincerely the numerical values that relate the shape to one of a kind of physical residences, artificial reactivity, and natural biological activities [21,22].

Loads of research has been executed inside the course of M-polynomial, as in the case of Munir et al., processed M-polynomial and related lists of triangular boron nanotubes in [6], polyhex nanotubes in [23], nanostar dendrimers in [4], and titania nanotubes in [5]. M-Polynomials and topological lists of V-phenylenic nanotubes and nanotori. In this paper, the objective is to process the M-polynomial of the crystallographic realistic structure of the atom copper(I) oxide $\left(\mathrm{Cu}_{2} \mathrm{O}\right)[8,24]$.

1.2. Crystallographic Structure of $\mathrm{Cu}_{2} \mathrm{O}(m ; n)$. Copper oxide is a p-type semiconductor and inorganic compound. Copper oxide is a chemical element with formula $\mathrm{Cu}_{2} \mathrm{O}(m ; n)$. $\mathrm{Cu}_{2} \mathrm{O}(m ; n)$ is a certainly happening reddish coral that is particularly used in chemical sensors and solar orientated cells in $[8,24]$. It has many advantages such as photochemical effects, stability, pigment, a fungicide, nontoxicity, and low cost. It has potential applications in new energy, sensing, sterilization, and other fields. It has narrow band gap and is easily excited by visible light.

$\mathrm{Cu}_{2} \mathrm{O}(m ; n)$ is additionally responsible for the pink shading in Benedict's test and is the essential cause to select $\mathrm{Cu}_{2} \mathrm{O}$ (see Figures 1 and 2). The promising projects of $\mathrm{Cu}_{2} \mathrm{O}(m ; n)$ are mainly on chemical sensors, sunlight-based cells, photocatalysis, lithium particle batteries, and catalysis. Here, we have taken into consideration a monolayer of $\mathrm{Cu}_{2} \mathrm{O}(m ; n)$ for satisfaction. To ultimate the basis for $\mathrm{Cu}_{2} \mathrm{O}(m ; n)$, we pick out the setting of this graph as $\mathrm{Cu}_{2} \mathrm{O}(m$; $n)$ be the chemical graph of copper(I) oxide with $(m ; n)$ unit cells within the aircraft.

\section{Definitions and Literature Review}

2.1. M-Polynomial. M-Polynomial is defined by S. Klavžar or E. Deutsch in 2015 [3, 8]. Within the factors of degreebased topological indices, we compete necessary role of M-polynomial. Readers can refer to [9-17, 27-35]. It is the foremost general progressive polynomial and an additionally closed formula alongside 10 distance-based topological indices is given by M-polynomial. It is explained as

$$
M(G, a, b)=\sum_{\delta \leq i \leq j \leq \Delta} m_{i j}(G) a^{i} b^{J}
$$

and we have $\delta=\operatorname{Min}\left\{d_{r} \mid r \in V(G)\right\}$ and $\Delta=\operatorname{Max}\left\{d_{r} \mid r \in V\right.$ $(G)\}$, where $m_{i j}(G)$ is the edge $E(G)$, where $i \leq j$.

2.2. Degree-Based Topological Indices. Any purpose on a graph which does not build upon numbering of its vertices is molecular descriptor. This is also called as topological index. Topological indices are most useful in the field of isomeric discrimination, chemical validation, QSAR, QSPR, and a pharmaceutical drug form. Topological indices are accessed from the system of molecule.

There are some important degree-based topological indices defined, and the first Zagreb index was introduced by Gutman and Trinajstić as follows:

$$
M_{1}(G)=\sum_{r s \varepsilon E(G)}\left(d_{r}+d_{s}\right) .
$$

Gutman and Trinajstić proposed the second Zagreb index in 1972, which is stated as

$$
M_{2}(G)=\sum_{r s \varepsilon E(G)}\left(d_{r} \times d_{s}\right) .
$$

The second modified Zagreb index is defined as

$$
{ }^{m} M_{2}(G)=\sum_{r s \varepsilon E(G)} \frac{1}{d(r) d(s)} .
$$

General $1^{\text {st }}$ and $2^{\text {nd }}$ multiplicative Zagreb indices are introduced by Kulli, Stone, Wang, and Wei and are stated as

$$
\begin{aligned}
& M Z_{1}^{a} I I(G)=\prod_{r, s \in E(G)}\left(d_{r}+d_{s}\right)^{a}, \\
& M Z_{2}^{a} I I(G)=\prod_{r, s \in E(G)}\left(d_{r}+d_{s}\right)^{a} .
\end{aligned}
$$

The general $1^{\text {st }}$ and $2^{\text {nd }}$ Zagreb indices proposed by Kulli, Stone, Wang, and Wei are stated as

$$
\begin{aligned}
& Z_{1}^{a}(G)=\sum_{r, s \in E(G)}\left(d_{r}+d_{s}\right)^{a}, \\
& Z_{2}^{a}(G)=\sum_{r, s \in E(G)}\left(d_{r} d_{s}\right)^{a} .
\end{aligned}
$$

In 1987, Fajtlowicz in [36] proposed the harmonic index and stated

$$
H(G)=\sum_{r, s \in E(G)} \frac{2}{d_{r}+d_{s}} .
$$

The inverse sum index is defined:

$$
I(G)=\sum_{r, s \in E(G)} \frac{d_{r} d_{s}}{d_{r}+d_{s}}
$$

Symmetric division index is described as

$$
\text { SS } D(G)=\sum_{r, s \in E(G)} \frac{\min \left(d_{r}, d_{s}\right)}{\max \left(d_{r}, d_{s}\right)}+\frac{\max \left(d_{r}, d_{s}\right)}{\min \left(d_{r}, d_{s}\right)} \text {. }
$$

SU and XU recognized general Randić index or general multiplicative Randić index stated as follows (Table 1): 


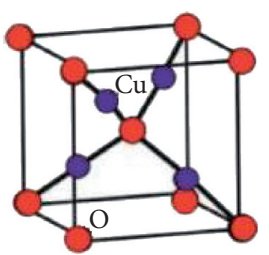

(a)

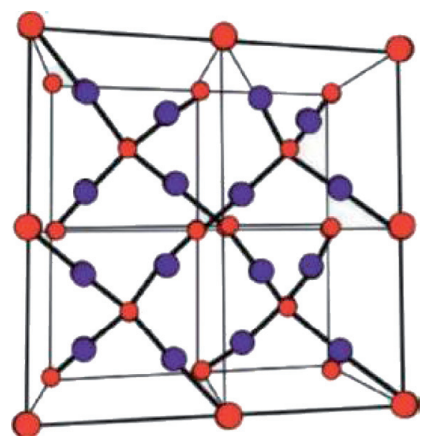

(b)

Figure 1: (a) $\mathrm{Cu}_{2} \mathrm{O}[1,1]$ [25]; (b) $\mathrm{Cu}_{2} \mathrm{O}[2,2]$ [1].

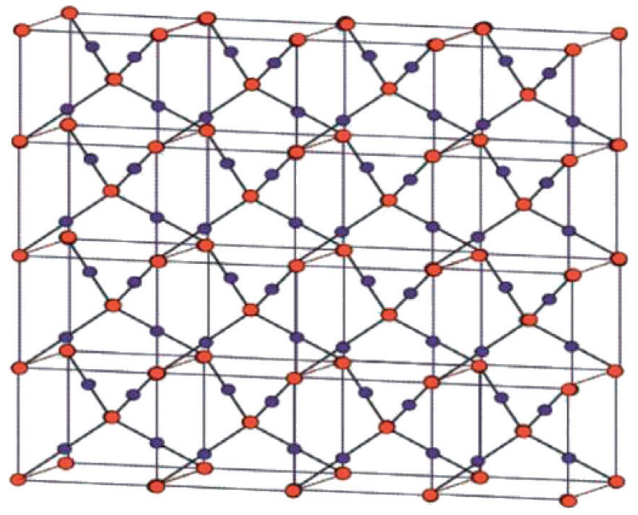

Figure 2: Copper(I) oxide [4, 4] [26].

$$
\begin{aligned}
R_{\alpha}(G) & =\sum_{r, s \in E(G)} d_{r}+d_{s}^{\alpha}, \\
R_{\alpha} I I(G) & =\prod_{r, s \in E(G)} d_{r}+d_{s}^{\alpha}
\end{aligned}
$$

Theorem 1. Crystallographic structure of the graph of cop$\operatorname{per}(I)$ oxide $G \approx \mathrm{Cu}_{2} \mathrm{O}[m ; n]$, where $n ; m \geq 1$. We have

$$
\begin{aligned}
M(G ; a ; b)= & f(a ; b)=(4 m+4 n-4) a b^{2} \\
& +(4 m n-4 n-4 m+4) a^{2} b^{2}+4 m n a^{2} b^{4}
\end{aligned}
$$

Proof. suppose $\mathrm{G}$ be the crystallographic structure of $\mathrm{Cu}_{2} \mathrm{O}$ $[l ; m ; n]$. The edge set of $\mathrm{Cu}_{2} \mathrm{O}[l ; m ; n]$ has the following three partitions by Figures 1 and 2:

$$
\begin{aligned}
& E_{1}=E_{\{1 ; 2\}}=\left\{e=r s \in E(G) \mid d_{r}=1 ; d_{s}=2\right\}, \\
& E_{2}=E_{\{2 ; 2\}}=\left\{e=r s \in E(G) \mid d_{r}=2 ; d_{s}=2\right\}, \\
& E_{3}=E_{\{2 ; 4\}}=\left\{e=r s \in E(G) \mid d_{r}=2 ; d_{s}=4\right\},
\end{aligned}
$$

such that

$$
\begin{aligned}
& \left|E_{1}(G)\right|=4 m m+4 n-4, \\
& \left|E_{2}(G)\right|=4 m n-4 m-4 n+4, \\
& \left|E_{3}(G)\right|=4 m n .
\end{aligned}
$$

Thus, the M-polynomial of $\mathrm{Cu}_{2} \mathrm{O}[l ; m ; n]$ is

$$
\begin{aligned}
& M(G, a, b)=\sum_{i \leq j} m_{i j}(G) a^{i} b^{j} \\
& M(G, a, b)=\sum_{1 \leq 2} m_{12}(G) a b^{2}+\sum_{2 \leq 2} m_{22}(G) a^{2} b^{2}+\sum_{2 \leq 4} m_{24}(G) a^{2} b^{4} \\
& M(G, a, b)=\sum_{r s \in E_{1}} m_{12}(G) a b^{2}+\sum_{u v \in E_{2}} m_{22}(G) a^{2} b^{2}+\sum_{u v \in E_{3}} m_{24}(G) a^{2} b^{4} \\
& M(G ; a ; b)=\left|E_{1}(G)\right| a b^{2}+\left|E_{2}(G)\right| a^{2} b^{2}+\left|E_{3}(G)\right| a^{2} y^{4} \\
& M(G ; a ; b)=(4 m+4 n-4) a b^{2}+(4 m n-4 n-4 m+4) a^{2} b^{2}+4 m n a^{2} b^{4}
\end{aligned}
$$

Theorem 2. Crystallographic structure of the graph of cop$\operatorname{per}(I)$ oxide $G \approx \mathrm{Cu}_{2} \mathrm{O}[m ; n]$, where $n ; m \geq 1$. We have $M_{1}(G)=40 m n-4 m-4 n+4$.

$$
\begin{aligned}
M(G ; a ; b)= & f(a ; b)=(4 m+4 n-4) \times a b^{2} \\
& +(4 m n-4 n-4 m+4) \times a^{2} b^{2}+4 m n \times a^{2} b^{4} .
\end{aligned}
$$

We have to find 
TABLE 1: Formulas of degree-based topological indices from M-polynomial.

\begin{tabular}{lcc}
\hline Topological Indices & $f(t, s)$ & $M(G ; t, s)$ \\
\hline First Zagreb index & $t+s$ & $M_{1}(G ; t, s)=\left.\left(D_{t}+D_{s}\right) M(G ; t, s)\right|_{t=s=1}$ \\
Second Zagreb index & $T s$ & $M_{2}(G ; t, s)=\left.\left(D_{t} D_{s}\right) M(G ; t, s)\right|_{t=s=1}$ \\
Second modified Zagreb index & $1 / t s$ & $m_{2}(G ; t, s)=\left.\left(\delta_{t} \delta_{s}\right) M(G ; t, s)\right|_{t=s=1}$ \\
General Randić index, $\alpha \neq 0$ & $(t s)^{\alpha}$ & $R_{\alpha}(G)=\left.\left(D_{t}^{\alpha} D_{s}^{\alpha}\right) M(G ; t, s)\right|_{t=s=1}$ \\
Inverse general Randić index, $\alpha \neq 0$ & $1 /(t s)^{\alpha}$ & $\left.R R_{\alpha}(G)\left(\delta_{t}^{\alpha} \delta_{s}^{\alpha}\right) M(G ; t, s)\right|_{t=s=1}$ \\
Symmetric division index & $\left(t^{2}+s^{2}\right) / t s$ & $S S D(G)=\left|D_{t} \delta_{s}=\delta_{s} D_{t}\right|_{t=s=1}$ \\
Harmonic index & $2 /(t+s)$ & $H(G)=\left.2 \delta_{t} J M(G ; t, s)\right|_{t=1}$ \\
Inverse sum index & $t s /(t+s)$ & $I(G)=\left.\delta_{t} J D_{t} D_{s} M(G ; t, s)\right|_{t=1}$ \\
\hline
\end{tabular}

$D_{s}=s(\partial / \partial s) M(G ; t, s)\left|t=s=1, D_{t}=t(\partial / \partial t) M(G ; t, s)\right| t=s=1, \delta_{t}=\int_{0}^{t}(M(G ; y, s)) / y \mathrm{~d} t, \delta_{s}=\int_{0}^{t} M(G ; t, y) / y \mathrm{~d} s, J=M(G ; t, t), Q_{\alpha}=x^{\alpha} M(G ; t, s), \alpha \neq 0$.

$$
\begin{aligned}
& D_{a}=\frac{\partial f}{\partial a} a, \\
& \frac{\partial f}{\partial a}=(4 n+4 m-4) b^{2}+2(4 m n-4 n-4 m+4) a b^{2}+8 m n a b^{4}
\end{aligned}
$$

Multiply $a$ on both sides:

$$
\begin{aligned}
D_{a}= & a \frac{\partial f}{\partial a}=(4 m+4 n-4) a b^{2} \\
& +2(4 m n-4 m-4 n+4) a^{2} b^{2}+8 m n a^{2} b^{4} .
\end{aligned}
$$

Similarly,

$$
\begin{aligned}
D_{b} f(a, b)= & b \frac{\partial f}{\partial b}=2(4 n+4 m-4) a b^{2} \\
& +2(4 m n-4 n-4 m+4) a^{2} b^{2}+16 m n a b^{4} \\
M_{1}(G)= & \left.\left(D_{a}+D_{y}\right) f(a, b)\right|_{a=b=1} .
\end{aligned}
$$

Now, the first Zagreb index is

$$
\begin{aligned}
M_{1}(G)= & \left.\left(D_{a}+D_{y}\right) f(a ; b)\right|_{a=b=1}, \\
M_{1}(G)= & {[(4 m+4 n-4)+2(4 m n-4 m-4 n+4)} \\
& +8 m n]+[2(4 n+4 m-4)] \\
& +2(4 m n-4 m-4 n+4)+16 m n), \\
M_{1}(G)= & {[4 m+4 n-4+8 m n-8 m-8 n+8+8 m n} \\
& +8 n+8 m-8+8 m n-8 m-8 n+8+16 m n] .
\end{aligned}
$$

After solving, the result is

$$
M(G)=40 m n-4 m-4 n+4 .
$$

The 3D plot of first Zagreb index is given in Figure 3 ( $f$ or $u=1$ left, $v=1$ middle, and $w=1$ right), and we see the dependent variables of the first Zagreb index on the involved parameters.

Theorem 3. Crystallographic structure of the graph of cop$\operatorname{per}(I)$ oxide $G \approx C u_{2} \mathrm{O}[m ; n]$, where $n ; m \geq 1$. We have $M_{2}(G)=48 m n-8 m-8 n+8$.
Proof. suppose

$$
\begin{aligned}
M(G ; a ; b)= & (4 m+4 n-4) \times a b^{2}+(4 m n-4 n-4 m+4) \\
& \times a^{2} b^{2}+4 m n \times a^{2} b^{4} .
\end{aligned}
$$

We have to find $D_{b} D_{a}$; first, we take $D_{a}$ :

$$
\begin{aligned}
D_{a}= & (4 m+4 n-4) \times a b^{2}+(4 m n-4 m-4 n+4)^{2} a \\
& \times a \times b^{2}+4 m n^{2} a \times a \times b^{4}, \\
D_{a}= & (4 m+4 n-4) \times a b^{2}+2(4 m n-4 m-4 n+4) \\
& \times a^{2} b^{2}+8 m n \times a^{2} b^{4} .
\end{aligned}
$$

Now, take $D_{b}$ :

$$
\begin{aligned}
D_{b} D_{a} f(a ; b)= & 2(4 m+4 n-4) a b \\
& +2(4 m n-4 m-4 n+4) a^{2} \times 2 b: b \\
& +8 m n a^{2} \times 4 b^{3} \times b, \\
D_{b} D_{a} f(a ; b)= & 2(4 m+4 n-4) \times a b^{2} \\
& +4(4 m n-4 m-4 n+4) \times a^{2} b^{2} \\
& +32 m n \times a^{2} b^{4} .
\end{aligned}
$$

The second Zagreb index is

$$
\begin{aligned}
& M_{2}(G)=\left.D_{b} D_{a}(f(a, b))\right|_{a=b=1}, \\
& M_{2}(G)=2(4 m+4 n-4)+4(4 m n-4 m-4 n+4)+32 m n, \\
& M_{2}(G)=8 n n+8 m-8+16 m n-16 m-16 n+16+32 m n .
\end{aligned}
$$

After solving, the result is

$$
M_{2}(G)=48 m n-8 m-8 n+8 .
$$

The $3 \mathrm{D}$ plot of second Zagreb index is given in Figure $4(f$ or $u=1$ left, $v=1$ middle, and $w=1$ right), and we see the dependent variables of the second Zagreb index on the involved parameters.

Theorem 4. Crystallographic structure of the graph of cop$\operatorname{per}(I)$ oxide $G \approx C u_{2} O[m ; n]$, where $n ; m \geq 1$, and we have

$$
{ }^{m} M^{2}(G)=\frac{3}{2} m n+m+n-1.3 \text {. }
$$




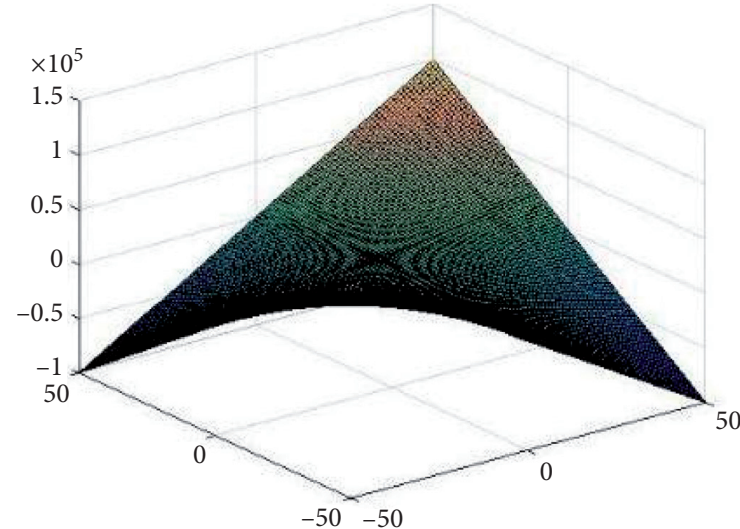

(a)

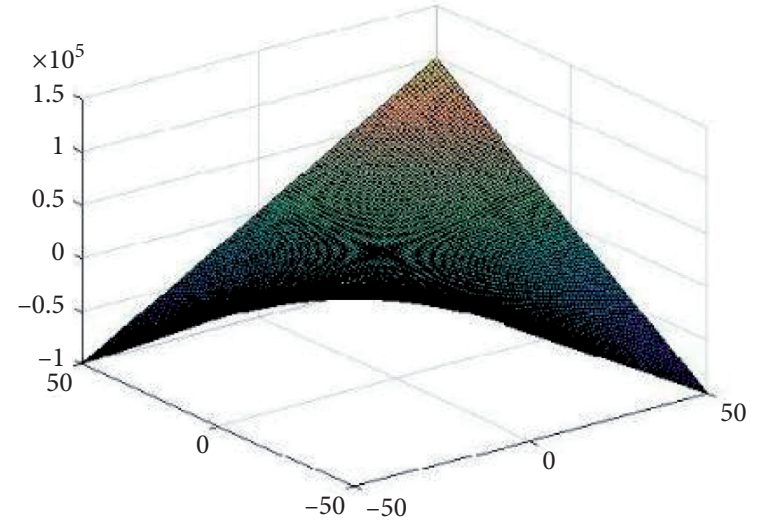

(b)

Figure 3: First Zagreb index plotted in 3D.

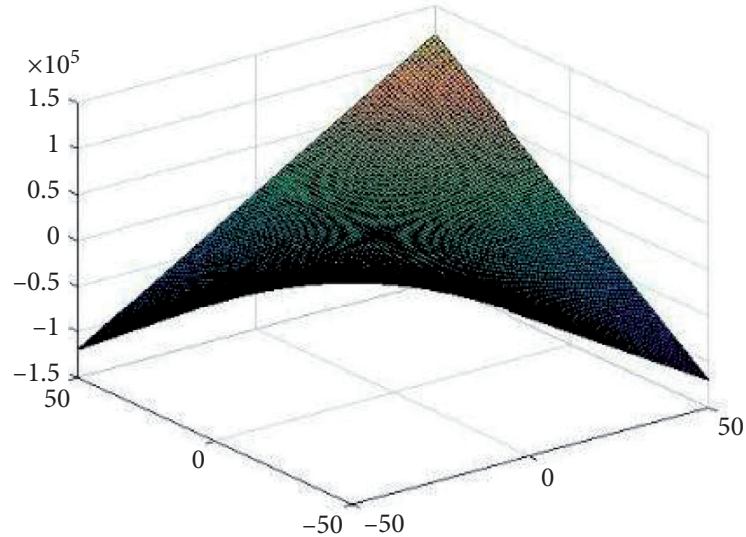

(a)

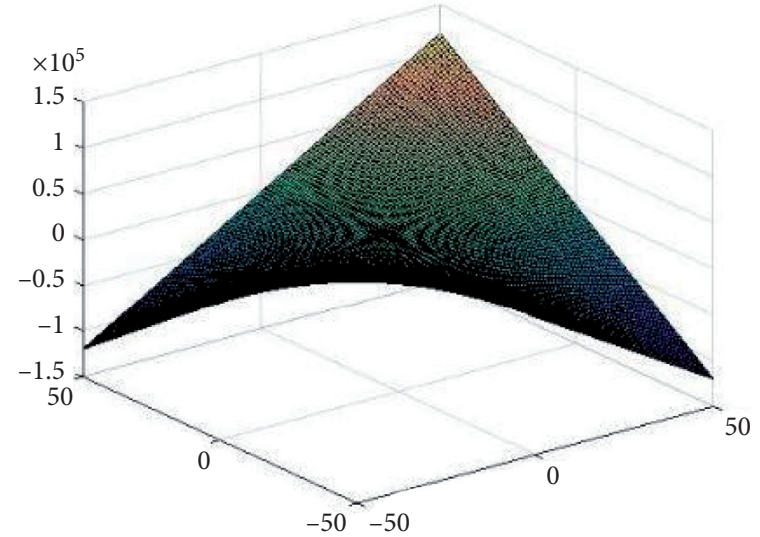

(b)

FIgURE 4: Second Zagreb index plotted in 3D.

Proof. suppose

$$
\begin{aligned}
M(G ; a ; b)= & (4 m+4 n-4) a b^{2} \\
& +(4 m n-4 m-4 n+4) a^{2} b^{2}+4 m n a^{2} b^{4}
\end{aligned}
$$

Now, we have to find $S_{a} S_{b}$; first, we find $S_{a}$ :

$$
\begin{aligned}
S_{a}= & \int_{0}^{a} \frac{f(x, b)}{x} \mathrm{~d} x, \\
f(x, b)= & (4 n+4 m-4) x b^{2}+(4 m n-4 m-4 n+4) x^{2} b^{2} \\
& +4 m n x^{2} b^{4} \\
\frac{f(x, b)}{x}= & (4 m+4 n-4) b^{2}+(4 m n-4 n-4 m+4) x b^{2} \\
& +4 m n x b^{4} .
\end{aligned}
$$

Taking integration on both sides,

$$
\begin{aligned}
\int_{0}^{a} \frac{f(x, b)}{x} \mathrm{~d} x= & \int_{0}^{a}(4 m+4 n-4) b^{2} d x \\
& +\int_{0}^{a}(4 m n-4 m-4 n+4) x b^{2} \mathrm{~d} x \\
& +4 m n \int_{0}^{a} x \mathrm{~d} x b^{4} \\
S_{a}= & (4 m+4 n-4) a b^{2} \\
& +\frac{1}{2}(4 m n-4 n-4 m+4) a^{2} b^{2}+2 m n a^{2} b^{4} .
\end{aligned}
$$

Now, take $S_{b}$ and then 
$S_{a} S_{b} f(a, b)=(4 m+4 n-4) a x^{2}$ $+\frac{1}{2}(4 m n-4 m-4 n+4) a^{2} x^{2}+2 m n a^{2} x^{4}$

$S_{a} S_{b} f(a, b)=\frac{1}{2}(4 m+4 n-4) a b^{2}$ $+\frac{1}{4}(4 m n-4 m-4 n+4) a^{2} b^{2}+\frac{1}{2} m n a^{2} b^{4}$.
Now, the second modified Zagreb index is

$$
\begin{aligned}
{ }^{m} M_{2}(G)=\left.S_{a} S_{b} f(a, b)\right|_{a=b=1} & =\frac{1}{2}(4 m+4 n-4)+\frac{1}{4}(4 m n-4 m-4 n+4)+\frac{1}{2} m n \\
& =(2 m+2 n-2)+(m n-m-n+1)+\frac{1}{2} m n \\
& =2 m-m+2 n-n-2+1+m n\left(1+\frac{1}{2}\right) .
\end{aligned}
$$

After solving, the result is

$$
{ }^{m} M_{2}(G)=\frac{3}{2} m n+m+n-1 .
$$

The 3D plot of modified second Zagreb index is given in Figure 5 ( $f$ or $u=1$ left, $v=1$ middle, and $w=1$ right), and we see the dependent variables of the modified second Zagreb index on the involved parameters.

Theorem 5. Crystallographic structure of the graph of cop$\operatorname{per}(I)$ oxide $G \approx C u_{2} O[m ; n]$, where $n ; m \geq 1$, and we have

$$
R_{\alpha}(G)=\left(2^{\alpha+2}-2^{2 \alpha+2}\right)(m+n-1)+\left(2^{2 \alpha+2}+2^{3 \alpha+2}\right) m n .
$$

Proof. suppose

$$
\begin{aligned}
M(G ; a ; b)= & (4 m+4 n-4) \times a b^{2}+(4 m n-4 m-4 n+4) \\
& \times a^{2} b^{2}+(4 m n) \times a^{2} b^{4}
\end{aligned}
$$

We have to find $D_{a} D_{b}$ first, and we find $D_{a}$ :

$$
\begin{aligned}
D_{a}= & (4 m+4 n-4) \times a b^{2}+2(4 m n-4 m-4 n+4) \\
& \times a^{2} b^{2}+8 m n \times a^{2} b^{4} .
\end{aligned}
$$

Now, take $D_{b}$ :

$$
\begin{aligned}
D_{a} D_{b}= & (4 m+4 n-4) a \times 2 b \times b+2(4 m n-4 n-4 m+4) a^{2} \\
& \times 2 b \times b+2(4 m n) a^{2} \times 4 b^{3} \times b .
\end{aligned}
$$

$$
\begin{aligned}
D_{a}^{\alpha} D_{b}^{\alpha}= & 2^{\alpha}(4 m+4 n-4) a b^{2}+4^{\alpha}(4 m n-4 m-4 n+4) a^{2} b^{2} \\
& +8^{\alpha}(4 m n) a^{2} b^{4} \\
D_{a}^{\alpha} D_{b}^{\alpha}= & 2^{\alpha+2} m+n-1 a b^{2}+2^{2 \alpha+2}(m n-m-n+1) a^{2} b^{2} \\
& +2^{3 \alpha+2} m n a^{2} b^{4} .
\end{aligned}
$$

Now, the general Randić index is

$$
\begin{aligned}
R_{\alpha}(G)= & \left.D_{a}^{\alpha} D_{b}^{\alpha}(f(a, b))\right|_{a=b=1}, \\
R_{\alpha}(G)= & 2^{\alpha+2}(m+n-1)+2^{2 \alpha+2}(m n-m-n+1)+2^{3 \alpha+2} m n, \\
R_{\alpha}(G)= & 2^{\alpha+2} m+2^{\alpha+2} n-2^{\alpha+2}+2^{2 \alpha+2} m n-2^{2 \alpha+2} m \\
& -2^{2 \alpha+2} n+2^{2 \alpha+2}+2^{3 \alpha+2} m n .
\end{aligned}
$$

The result is

$$
R_{\alpha}(G)=\left(2^{\alpha+2}-2^{2 \alpha+2}\right)(m+n-1)+\left(2^{2 \alpha+2}+2^{3 \alpha+2}\right) m n .
$$

The 3D plot of Randić index is given in Figure 6 ( $f$ or $u=1$ left, $v=1$ middle, and $w=1$ right), and we see the dependent variables of the Randić index on the involved parameters.

Theorem 6. Crystallographic structure of the graph of cop$\operatorname{per}(I)$ oxide $G \approx C u_{2} 0[m ; n]$, where $n ; m \geq 1$, and we have

$$
\begin{aligned}
R R_{\alpha}(G)= & {\left[\frac{1}{2^{\alpha-2}}-\frac{1}{2^{2 \alpha-2}}\right](m+n)+\left[\frac{1}{2^{2 \alpha-2}}+\frac{2}{2^{3 \alpha-2}}\right](m n) } \\
& +\left[\frac{1}{2^{\alpha-2}}+\frac{1}{2^{2 \alpha-2}}\right] .
\end{aligned}
$$

Take $\alpha$ on the above equation: 


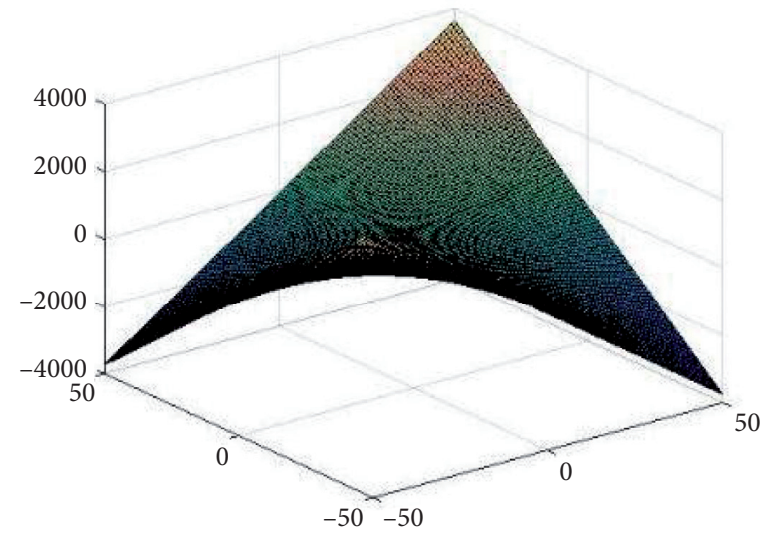

(a)

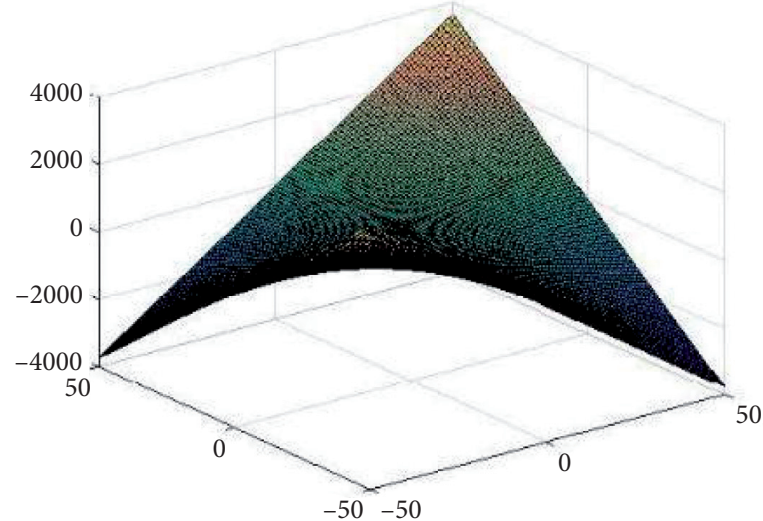

(b)

Figure 5: Modified the second Zagreb index plotted in 3D.

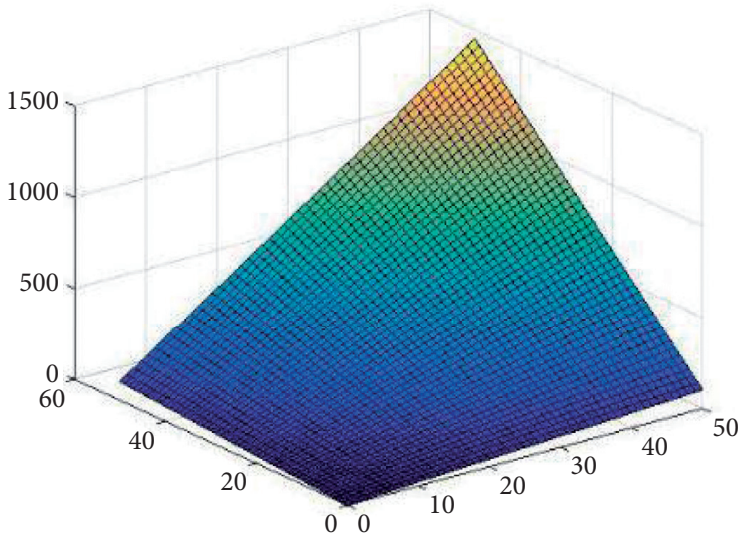

(a)

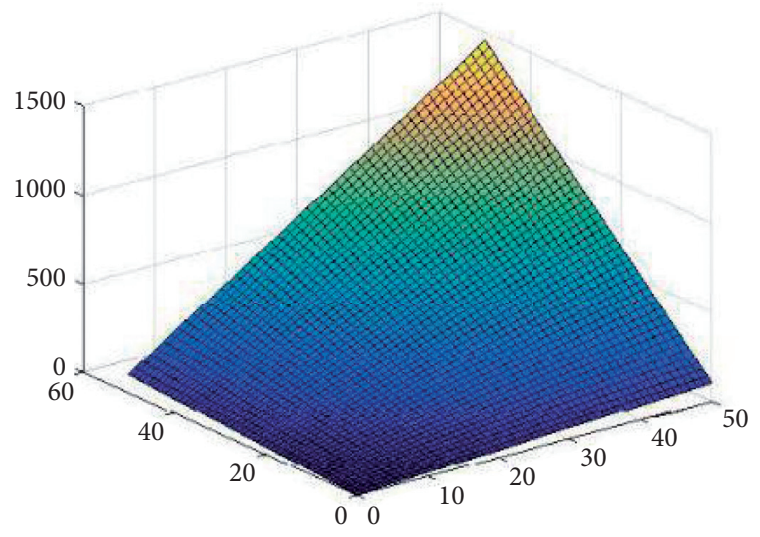

(b)

FIgURE 6: Randić index plotted in 3D.

Proof. suppose

$$
\begin{aligned}
M(G ; a ; b)= & (4 m+4 n-4) \times a b^{2}+(4 m n-4 m-4 n+4) \\
& \times a^{2} b^{2}+(4 m n) \times a^{2} b^{4}
\end{aligned}
$$

Now, we have to find $S_{a} S_{b}$, and first, we find $S_{a}$ :

$$
\begin{aligned}
S_{a}= & (4 m+4 n-4) \int_{0}^{a} \mathrm{~d} x \cdot b^{2}+(4 m n-4 m-4 n+4) \\
& \cdot \int_{0}^{a} x \mathrm{~d} x \cdot b^{2}+8 m n \int_{0}^{a} x \mathrm{~d} x \cdot b^{4} \\
S_{a}= & (4 m+4 n-4) a b^{2}+2(m n-m-n+1) a^{2} b^{2} \\
& +4 m n a^{2} b^{4} .
\end{aligned}
$$

$$
\begin{aligned}
S_{a} S_{b}= & 4(m+n-1) a \cdot \int_{0}^{b} x \mathrm{~d} x+2(m n-n-m+1) a^{2} \\
& \cdot \int_{0}^{b} x \mathrm{~d} x+4 m n a^{2} \int_{0}^{b} x^{3} \mathrm{~d} x \\
S_{a} S_{b}= & 2(m+n-1) a b^{2}+(m n-m-n+1) a^{2} b^{2} \\
& +m n a^{2} b^{4} .
\end{aligned}
$$

Take $\alpha$ on the above equation:

$$
\begin{aligned}
S_{a}^{\alpha} S_{b}^{\alpha}= & \frac{1}{2^{\alpha-2}}(m+n-1) a b^{2}+\frac{1}{2^{2 \alpha-2}}(m n-m-n+1) a^{2} b^{2} \\
& +\frac{1}{2^{3 \alpha-2}} m n a^{2} b^{4} .
\end{aligned}
$$

Similarly, take $S_{b}$ :
The inverse Randić is 


$$
\begin{aligned}
R R_{\alpha}(G)= & \left.(f(a, b))\right|_{a=b=1}=\frac{1}{2^{\alpha-2}}(m+n-1) \\
& +\frac{1}{2^{2 \alpha-2}}(m n-m-n+1) \\
+\frac{2}{2^{3 \alpha-2}} m n= & {\left[\frac{1}{2^{\alpha-2}}-\frac{1}{2^{2 \alpha-2}}\right](m+n) } \\
& +\left[\frac{1}{2^{2 \alpha-2}}+\frac{2}{2^{3 \alpha-2}}\right](m n)+\left[\frac{1}{2^{\alpha-2}}+\frac{1}{2^{2 \alpha-2}}\right] .
\end{aligned}
$$

The 3D plot of inverse Randić index is represented in Figure 7 ( $f$ or $u=1$ left, $v=1$ middle, and $w=1$ right), and we see the dependent variables of the inverse Randic index on the involved parameters.

Theorem 7. Crystallographic structure of the graph of cop$\operatorname{per}(I)$ oxide $G \approx C u_{2} \mathrm{O}[m ; n]$, where $n ; m \geq 1$, and we have SS $D(G)=18 m n+2 m+2 n-2$.

Proof. suppose

$$
\begin{aligned}
M(G ; a ; b)= & (4 m+4 n-4) \times a b^{2}+(4 m n-4 m-4 n+4) \\
& \times a^{2} b^{2}+(4 m n) \times a^{2} b^{4} .
\end{aligned}
$$

First, we have to find $S_{b}$ :

$$
\begin{aligned}
S_{b}= & (4 n+4 m-4) a \int_{0}^{b} x \mathrm{~d} x+(4 m n-4 m-4 n+4) a^{2} \\
& \cdot \int_{0}^{b} x \mathrm{~d} x+4 m n a^{2} \int_{0}^{b} x^{3} \mathrm{~d} x, \\
S_{b}= & \frac{1}{2}(4 n+4 m-4) a b^{2}+\frac{1}{2}(4 m n-4 m-4 n+4) a^{2} b^{2} \\
& +m n a^{2} b^{4} .
\end{aligned}
$$

Now, take $D_{a}$ :

$S_{b} D_{a}=\frac{1}{2}(4 m+4 n-4) a b^{2}+(4 m n-4 m-4 n+4) a^{2} b^{2}+2 m n a^{2} b^{4}$.

Similarly,

$$
S_{a}=(4 m+4 n-4) a b^{2}+\frac{1}{2}(4 m n-4 n-4 m+4) a^{2} b^{2}+2 m n a^{2} b^{4} .
$$

Take $D_{b}$ :

$$
\begin{aligned}
S_{a} D_{b}(f(a, b))= & 2(4 m+4 n-4) a b^{2} \\
& +(4 m n-4 m-4 n+4) a^{2} b^{2}+8 m n a^{2} b^{4}
\end{aligned}
$$

Now, the symmetric division index is

$$
\text { SS } D(G)=\left.\left(S_{b} D_{a}+S_{a} D_{b}\right)(f(a, b))\right|_{a=b=1} \text {. }
$$

Put the values

$$
\begin{aligned}
& \text { SS } D(G)=\left[\frac{1}{2}(4 m+4 n-4)+(4 m n-4 m-4 n+4)+2 m n\right]+[2(4 m+4 n-4)+(4 m n-4 m-4 n+4)+8 m n] \\
& \text { SS } D(G)=(2 m+2 n-2)+(4 m n-4 m-4 n+4)+(2 m n+8 m+8 n-8)+(4 m n-4 m-4 n+4)+8 m n \\
& \text { SS } D(G)=(2 m-4 m+8 m-4 m)+(2 n-4 n+8 n-4 n)-(2-4+8-8)+(4 m n+2 m n+4 m n+8 m n) .
\end{aligned}
$$

After the calculation, the result is

$$
\text { SS } D(G)=18 m n+2 m+2 n-2 \text {. }
$$

The 3D plot of symmetric division index is given in Figure 8 ( $f$ or $u=1$ left, $v=1$ middle, and $w=1$ right), and we see the dependent variables of the symmetric division index on the involved parameters.

Theorem 8. Crystallographic structure of the graph of cop$\operatorname{per}(I)$ oxide $G \approx \mathrm{Cu}_{2} \mathrm{O}[m ; n]$, where $n ; m \geq 1$, and we have

$$
H(G)=\frac{5}{3}(m+n-1)+\frac{7}{3} m n \text {. }
$$

Proof. suppose

$$
\begin{aligned}
M(G ; a ; b)= & (4 m+4 n-4) \times a b^{2}+(4 m n-4 m-4 n+4) \\
& \times a^{2} b^{2}+(4 m n) \times a^{2} b^{4}
\end{aligned}
$$

First, we have to find $J_{f}(a ; b)$ :

$$
\begin{aligned}
J f(a, b)= & J f(a, a)=4(m+n-1) a^{3} \\
& +4(m n-m-n+1) a^{4}+8 m n a^{6} .
\end{aligned}
$$

Take $S_{a}$ : 


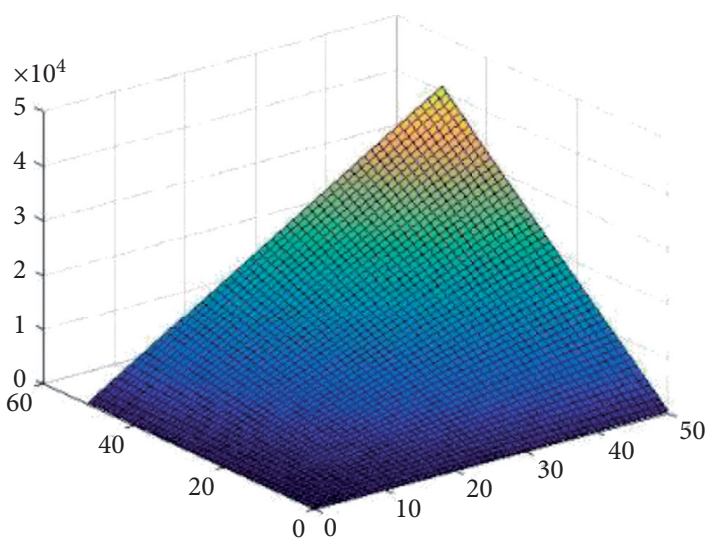

(a)

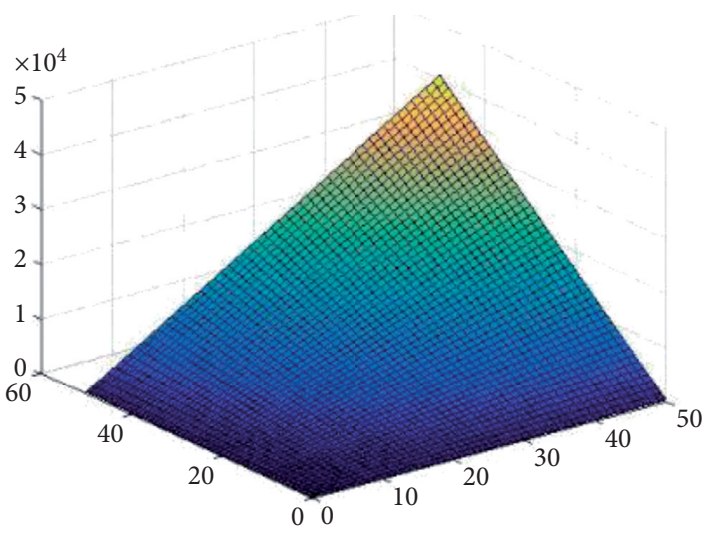

(b)

Figure 7: Inverse Randić index plotted in 3D.

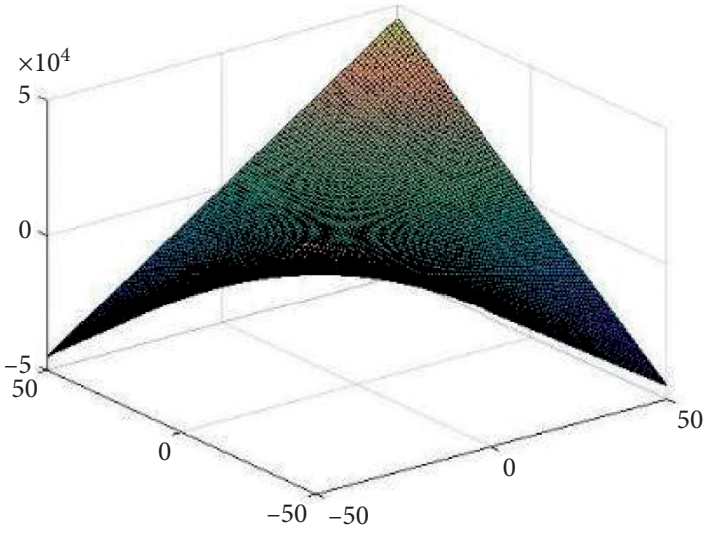

(a)

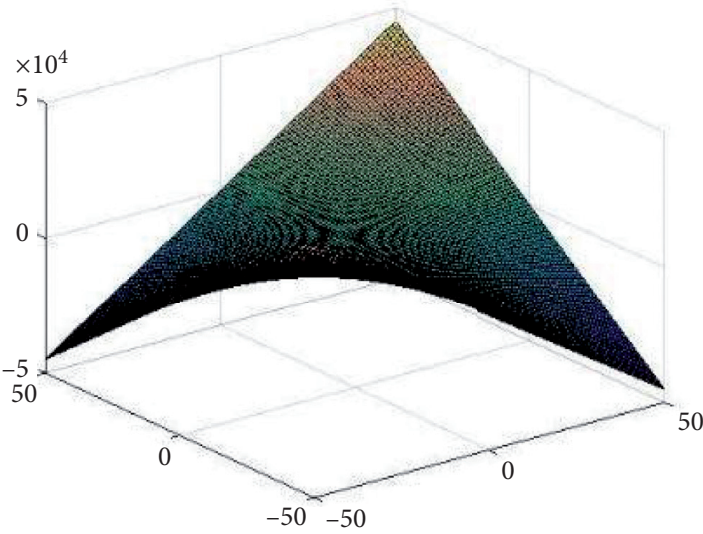

(b)

Figure 8: Symmetric division index plotted in 3D.

$$
\begin{aligned}
S_{a} J f(x, b)= & 4(m+n-1) \int_{0}^{a} x^{2} \mathrm{~d} x+4(m n-m-n+1) \\
& \cdot \int_{0}^{a} x^{3} \mathrm{~d} t+8 m n \int_{0}^{a} x^{5} \mathrm{~d} x, \\
S_{a} J f(a, b)= & \frac{4}{3}(m+n-1) a^{3}+\frac{1}{2}(m n-m-n+1) a^{4} \\
& +\frac{2}{3} m n a^{6} .
\end{aligned}
$$

The harmonic index is

$$
\begin{aligned}
H(G) & =\left.2 S_{a} J f(a, b)\right|_{a=1} \\
& =2\left[\frac{4}{3}(m+n-1)+\frac{1}{2}(m n-m-n+1)+\frac{2}{3} m n\right], \\
H(G) & =2\left[\left(\frac{4}{3}-\frac{1}{2}\right) m+\left(\frac{4}{3}-\frac{1}{2}\right) n+\left(\frac{1}{2}-\frac{4}{3}\right)+\left(\frac{4}{3}+\frac{1}{2}\right)\right] m n, \\
H(G) & =2\left[\frac{5}{6} m+\frac{5}{6} n+\frac{7}{6} m n-\frac{5}{6}\right] .
\end{aligned}
$$

Now, the result is

$$
H(G)=\frac{5}{3}(m+n-1)+\frac{7}{3} m n .
$$

The 3D plot of harmonic index is given in Figure 9 ( $f$ or $u=1$ left, $v=1$ middle, and $w=1$ right), and we see the dependent variables of the harmonic index on the involved parameters.

Theorem 9. Crystallographic structure of the graph of cop$\operatorname{per}(I)$ oxide $G \approx C u 20[m ; n]$, where $n ; m \geq 1$, and we have

$$
S_{a} J D_{a} D_{b}(f(a, b))=\frac{44}{3} m n-\frac{4}{3}(m+n-1) .
$$

Proof. suppose

$$
\begin{aligned}
M(G ; a ; b)= & (4 m+4 n-4) \times a b^{2}+(4 m n-4 m-4 n+4) \\
& \times a^{2} b^{2}+(4 m n) \times a^{2} b^{4} .
\end{aligned}
$$

(58) First, we have to find $D_{b}$ : 


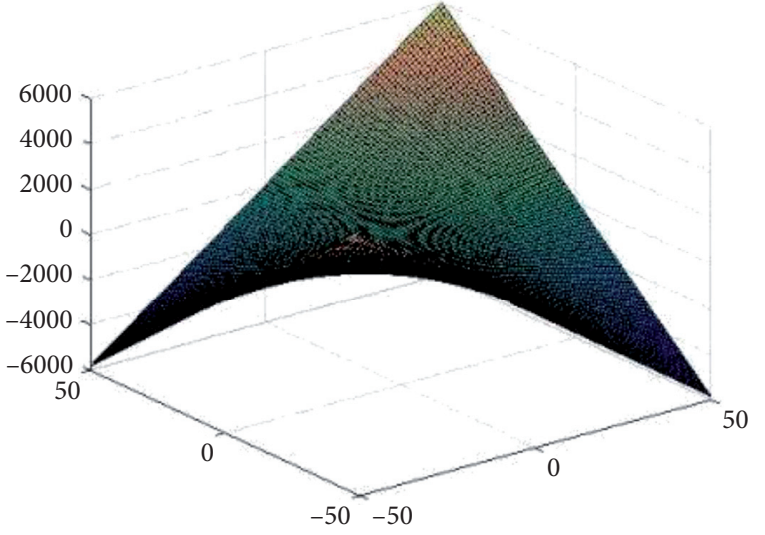

(a)

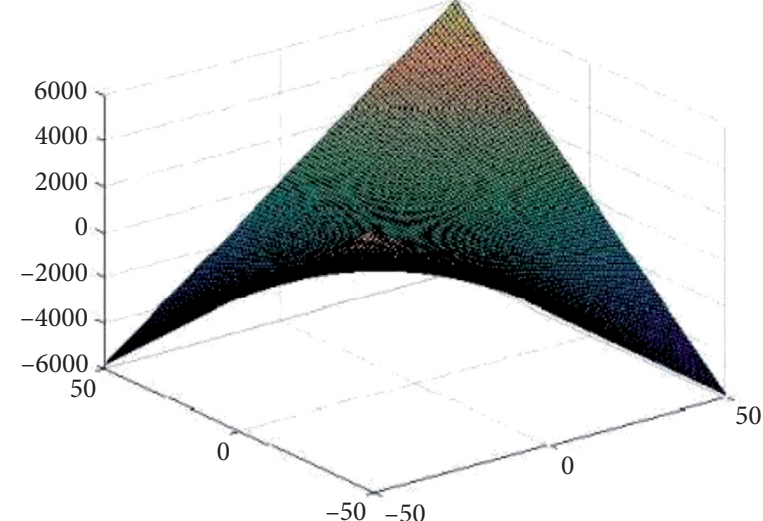

(b)

Figure 9: Harmonic index plotted in 3D.

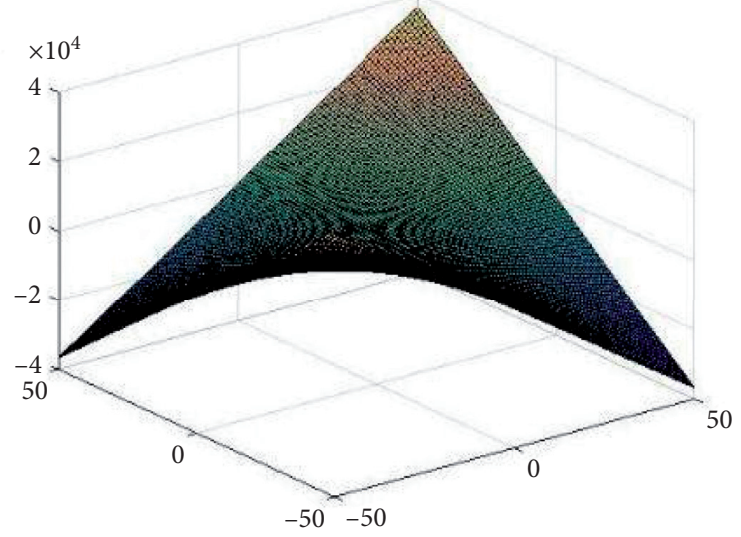

(a)

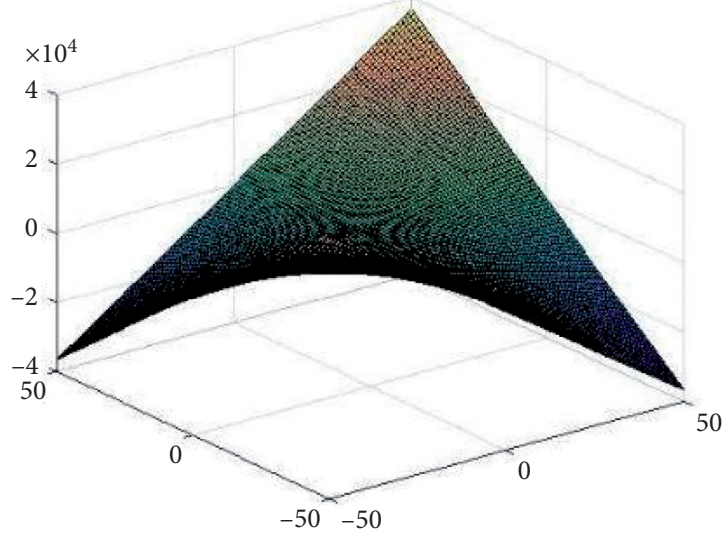

(b)

Figure 10: Inverse sum index plotted in 3D.

$$
\begin{aligned}
D_{b} f(a, b)= & 8(m+n-1) a b^{2}+8(m n+m+n-1) a^{2} b^{2} \\
& +32 m n a^{2} b^{4} .
\end{aligned}
$$

Take $D_{a}$ :

$$
\begin{aligned}
D_{a} D_{b} f(a, b)= & 8(m+n-1) a b^{2}+16(m n-n-m+1) a^{2} b^{2} \\
& +64 m n a^{2} b^{4} .
\end{aligned}
$$

Take $J_{f}(a ; b)$ :

$$
\begin{aligned}
J D_{a} D_{b} f(a, b)= & 8(m+n-1) x^{3}+16(m n-m-n+1) x^{4} \\
& +64 m n x^{6}
\end{aligned}
$$

$$
\begin{aligned}
S_{a} J D_{a} D_{b} f(a, b)= & \frac{8}{3}(m+n-1) a^{3} \\
& +4(m n-n-m+1) a^{4}+\frac{32}{3} m n a^{6} .
\end{aligned}
$$

The inverse sum index is

$$
\left.S_{a} J D_{a} D_{b}(f(a, b))\right|_{a=1}=\frac{8}{3}(m+n-1)
$$

$$
\begin{aligned}
& +4(m n-m-n+1)+\frac{32}{3} m n \\
= & \left(\frac{8}{3}-4\right) m+\left(\frac{8}{3}-4\right) n \\
& +\left(\frac{32}{3}+4\right) m n+\left(4-\frac{8}{3}\right) .
\end{aligned}
$$

Take $S(a)$ : 
After the calculation, the result is

$$
S_{a} J D_{a} D_{b}(f(a, b))=\frac{44}{3} m n-\frac{4}{3}(m+n-1) .
$$

The 3D plot of inverse sum index is given in Figure $10(f$ or $u=1$ left, $v=1$ middle, and $w=1$ right), and we see the dependent variables of the inverse sum index on the involved parameters.

\section{Data Availability}

No data were used in this study.

\section{Disclosure}

All authors have not any fund, grant, and sponsor for supporting publication charges.

\section{Conflicts of Interest}

The authors declare that there are no conflicts of interest regarding the publication of this paper.

\section{References}

[1] A. Q. Baig, M. Naeem, M. Naeem, W. Gao, and J.-B. Liu, "General fifth M-Zagreb indices and fifth M-Zagreb polynomials of carbon graphite," Eurasian Chemical Communications, vol. 2, no. 3, pp. 634-640, 2020.

[2] F. A. Cotton, G. Wilkinson, C. A. Murillo, and M. Bochmann, Advanced Inorganic Chemistry, John Wiley \& Sons, Hoboken, NJ, USA, 6th edition, 1999.

[3] H. Deng, J. Yang, and F. Xia, "A general modeling of some vertex-degree based topological indices in benzenoid systems and phenylenes," Computers \& Mathematics with Applications, vol. 61, no. 10, pp. 3017-3023, 2011.

[4] W. Gao, Y. Wang, B. Basavanagoud, and M. K. Jamil, "Characteristics studies of molecular structures in drugs," Saudi Pharmaceutical Journal, vol. 25, no. 4, pp. 580-586, 2017.

[5] G. Gayathri, U. Priyanka, and U. Priyanka, "Degree based topological indices of zig zag chain," Journal of Mathematics and Informatics, vol. 11, pp. 83-93, 2017.

[6] I. Gutman, "Some properties of the Winer polynomial," Graph Theory Notes N. Y.vol. 125, pp. 13-18, 1993.

[7] L. B. Kier and L. H. Hall, Molecular Connectivity in StructureActivity Analysis, Wiley, New York, NY, USA, 1986.

[8] X. Li and Y. Shi, "A survey on the Randic index," MATCH Communications in Mathematical and in Computer Chemistry, vol. 59, pp. 127-156, 2008.

[9] M. Alaeiyan, C. Natarajan, C. Natarajan, G. Sathiamoorthy, and M. R. Farahani, "The eccentric connectivity index of polycyclic aromatic hydrocarbons (PAHs)," Eurasian Chemical Communications, vol. 2, no. 6, pp. 646-651, 2020.

[10] M. Cancan, S. Ediz, H. Mutee-Ur-Rehman, and D. Afzal, "Mpolynomial and topological indices Poly (EThyleneAmidoAmine) dendrimers," Journal of Information and Optimization Sciences, vol. 41, no. 4, pp. 1117-1131, 2020.

[11] M. Cancan, S. Ediz, S. Ediz, and M. R. Farahani, "On vedegree atom-bond connectivity, sum-connectivity, geometric-arithmetic and harmonic indices of copper oxide," Eurasian Chemical Communications, vol. 2, no. 5, pp. 641-645, 2020.
[12] M. Imran, S. A. Bokhary, S. A. U. h. Bokhary, S. Manzoor, and M. K. Siddiqui, "On molecular topological descriptors of certain families of nanostar dendrimers," Eurasian Chemical Communications, vol. 2, no. 6, pp. 680-687, 2020.

[13] M. R. Farahani, "Some connectivity indices and Zagreb index of polyhex nanotubes," Acta Chimica Slovenica, vol. 59, pp. 779-783, 2012.

[14] M. Randic, "Characterization of molecular branching," Journal of the American Chemical Society, vol. 97, no. 23, pp. 6609-6615, 1975.

[15] B. Zhou and I. Gutman, "Relations between wiener, hyperwiener and Zagreb indices," Chemical Physics Letters, vol. 394, no. 1-3, pp. 93-95, 2004.

[16] Z. Ahmad, M. Naseem, M. Naseem, M. K. Jamil, M. F. Nadeem, and S. Wang, "Eccentric connectivity indices of titania nanotubes $\mathrm{TiO}_{2}[m ; n]$," Eurasian Chemical Communications, vol. 2, no. 6, pp. 712-721, 2020.

[17] Z. Ahmad, M. Naseem, M. Naseem, M. Kamran Jamil, M. Kamran Siddiqui, and M. Faisal Nadeem, "New results on eccentric connectivity indices of V-Phenylenic nanotube," Eurasian Chemical Communications, vol. 2, no. 6, pp. 663671, 2020.

[18] J. B. Babujee and S. Ramakrishnan, "Topological indices and new graph structures," Applied Mathematical Sciences, vol. 6, no. 108, pp. 5383-5401, 2012.

[19] E. Deutsch and S. Klavžar, "M-polynomial and degree-based topological indices," Iranian Journal of Mathematical Chemistry, vol. 6, p. 93102, 2015.

[20] A. A. Dobrynin, R. Entringer, and I. Gutman, "Wiener index of trees: theory and applications," Acta Applicandae Mathematicae, vol. 66, no. 3, pp. 211-249, 2001.

[21] D. Dimitrov, "On structural properties of trees with minimal atom-bond connectivity index IV: solving a conjecture about the pendent paths of length three," Applied Mathematics and Computation, vol. 313, pp. 418-430, 2017.

[22] F. Afzal, M. A. Razaq, M. Abdul Razaq, D. Afzal, and S. Hameed, "Weighted entropy of penta chains graph," Eurasian Chemical Communications, vol. 2, no. 6, pp. 652$662,2020$.

[23] I. Gutman and B. Furtula, Recent Results in the Theory of Randić Index, University of Kragujevac, Kragujevac, Serbia, 2008.

[24] L. B. Kier and L. H. Hall, Molecular Connectivity in Chemistry and Drug Research, Academic Press, New York, NY, USA, 1976.

[25] M. S. Ahmad, W. Nazeer, S. M. Kang, M. Imran, and W. Gao, "Calculating degree-based topological indices of dominating David derived networks," Open Physics, vol. 15, no. 1, pp. 1015-1021, 2017.

[26] A. Q. Baig, M. Imran, W. Khalid, and M. Naeem, "Molecular description of carbon graphite and crystal cubic carbon structures," Canadian Journal of Chemistry, vol. 95, no. 6, p. $674,2017$.

[27] F. M. Brückler, T. Došlić, A. Graovac, and I. Gutman, "On a class of distance-based molecular structure descriptors," Chemical Physics Letters, vol. 503, no. 4-6, pp. 336-338, 2011.

[28] E. A. Ekimov, V. A. Sidorov, E. D. Bauer, N. N. Melnik, N. J. Curro, and J. D. Thompson, "Superconductivity in diamond," Nature, vol. 428, p. 5425, 2004.

[29] F. Afzal, S. Hussain, D. Afzal, and S. Razaq, "Some new degree based topological indices via M-polynomial," Journal of Information and Optimization Sciences, vol. 41, no. 4, pp. 1061-1076, 2020. 
[30] W. Gao, Z. Iqbal, M. Ishaq, R. Sarfraz, M. Aamir, and A. Aslam, "On eccentricity-based topological indices study of a class of porphyrin-cored dendrimers," Biomolecules, vol. 8, no. 3, p. 71, 2018.

[31] W. Gao, H. Wu, M. K. Siddiqui, and A. Q. Baig, "Study of biological networks using graph theory," Saudi Journal of Biological Sciences, vol. 25, no. 6, pp. 1212-1219, 2018.

[32] Y. Hu, X. Li, Y. Shi, T. Xu, and I. Gutman, "On molecular graphs with smallest and greatest zeroth-order general Randic index," MATCH Communications in Mathematical and in Computer Chemistry, vol. 54, p. 425, 2005.

[33] H. Yang and X. Zhang, "The independent domination numbers of strong product of two cycles," Journal of Discrete Mathematical Sciences and Cryptography, vol. 21, no. 7-8, pp. 1495-1507, 2018.

[34] O. Ivanciuc, "Chemical graphs, molecular matrices and topological indices in chemoinformatics and quantitative structure-activity relationships $\$$," Current Computer AidedDrug Design, vol. 9, no. 2, pp. 153-163, 2013.

[35] S. M. Kang, M. A. Zahid, A. u. R. Virk, W. Nazeer, and W. Gao, "Calculating the degree-based topological indices of dendrimers," Open Chemistry, vol. 16, no. 1, pp. 681-688, 2018.

[36] G. Caporossi, I. Gutman, P. Hansen, and L. Pavlović, "Graphs with maximum connectivity index," Computational Biology and Chemistry, vol. 27, no. 1, pp. 85-90, 2003. 